\title{
Fault-Tolerant Transmission Mechanism for IEEE 802.16e OFDMA Systems
}

\author{
Jenhui Chen and Syao-Syuan Liu \\ Department of Computer Science and \\ Information Engineering \\ Chang Gung University, Taoyuan, Taiwan. \\ jhchen@mail.cgu.edu.tw
}

\author{
Chih-Chieh Wang \\ Department of Electrical Engineering \\ Chang Gung University, Taoyuan, Taiwan. \\ tier@ms1.hinet.net
}

\begin{abstract}
The most challenging issue in worldwide interoperability for microwave access network technologies is to find a way to counteract the interference as well as solve the fading problems in transmission. Although IEEE 802.16e standard is proposed to tackle this difficult problem, there are still many external interferences, which may corrupt ongoing transmissions. To diminish this effect, this paper proposes a faulttolerant transmission mechanism (FTM) by adjusting the burst size, rearranging slot allocation, and comparing patterns to provide a reliable, secure, and quality transmission. Simulation results reveal that in an efficient transmission, the burst size has to be considered no more than 20 slots; it should not exceed $5 \%$ of the total frame space. Furthermore, FTM yields approximately $30 \%$ throughput and significantly raises the successful transmission probability compared to IEEE 802.16e transmission mechanism, while still being fully compatible with all frame-based WiMAX system.
\end{abstract}

\section{Keywords}

Fault-tolerant, OFDMA, reliability, robust, wireless

\section{INTRODUCTION}

In view of the demand of wireless access and high bandwidth transmissions, the fixed broadband wireless access (BWA) system, such as the local multipoint distribution service (LMDS), is proposed to offer multimedia services to a number of discrete subscriber sites. This is accomplished by using base stations (BSs) to provide network access services for subscriber sites, which are based on the IEEE 802.16e wireless metropolitan area networks (WMANs) standards [4]. They comprise the medium access control (MAC) layer and the physical (PHY) layer [14], that dominate the main

\footnotetext{
${ }^{*}$ This work was supported in part by the National Science Council, Taiwan, R.O.C., under contract NSC96-2221-E182-004.
}

part of BWA. In IEEE 802.16e, there are many types of the physical specification, such as single carrier (SC), single carrier access (SCA), orthogonal frequency-division multiplexing (OFDM), and orthogonal frequency-division multiple access (OFDMA) [4]. OFDM and OFDMA have the advantages of resisting interferences, providing high bandwidth, and supporting diverse modulations. Especially, the OFDMA combines two major transmission techniques: OFDM and the frequency-division multiple access (FDMA).

An OFDMA slot is based on OFDMA symbol structure, which varies within the upload link (UL) and the download link (DL). The structure includes the full usage of the subchannels (FUSC), the partial usage of subchannels (PUSC), the distributed subcarrier permutations, and the adjacent subcarrier permutation [7]. As IEEE $802.16 \mathrm{e}$ standard described, one slot is one subchannel by two OFDMA symbols for PUSC DL burst. OFDMA inherits inter-symbol interference (ISI) and inter-carrier interference (ICI) which are against internal interference and frequency selective fading. Therefore, IEEE 802.16e WMANs standard and digital video broadcasting return channel terrestrial (DVB-RCT) proposed OFDMA for broadband wireless multiple access systems.

However, ICI may seriously damage the orthogonal characteristic and increase the probability of ISI simultaneously [17]. These inevitable internal factors will reduce the quality of transmissions. In addition, it will also encounter unpredictable external interferences called "noise" [5, 8], which may be produced by temperature, humidity, an external electromagnetic wave, etc. [16]. In the IEEE 802.16e scheduled transmission, each mobile subscriber station (MSS) has its data stream which may be arranged in a profile or in some subchannels. These profiles or subchannels are randomly put into slots for transmission. Therefore, much transmission frame space is left empty; the profile will waste much time in transmission. These mechanisms cause serious carrier-to-interference-and-noise ratio (CINR) [12]. Therefore, the IEEE 802.16e mechanism cannot counteract the external interference nor solve the fading problems [4]. Although the IEEE $802.16 \mathrm{e}$ standard is proposed to solve this difficult problem by using automatic repeat-request (ARQ) or high speed automatic repeat-request (HARQ) retransmission mechanisms to keep a higher successful transmission probability, these mechanisms will waste a lot of system resource. Other methods, such as guard time, cyclic extension, smart code, and adaptive power control [7, 8, 15] were also proposed to solve these problems. But the improvements of these methods are insufficient and will cause a great deal of
QShine 2008, July 28-31, 2008, Hong Kong, Hong Kong. Copyright 2008 ICST ISBN 978-963-9799-26-4 DOI 10.4108/ICST.QSHINE2008.3932 


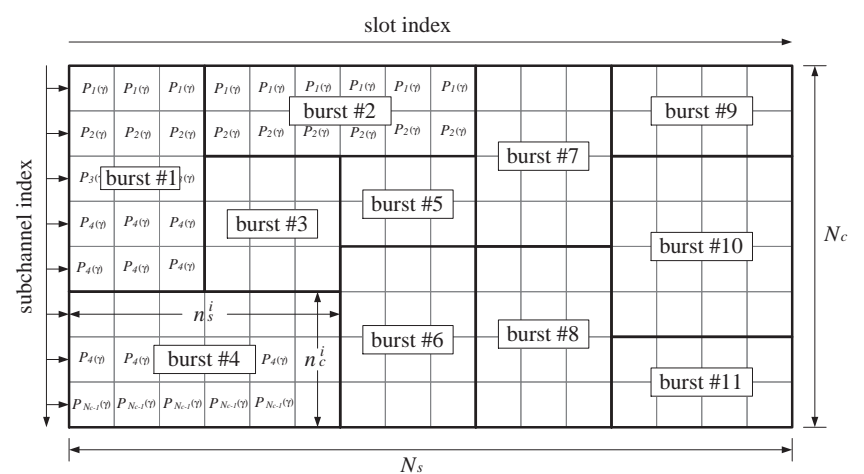

Figure 1: The diagram of the frame structure of IEEE 802.16e.

overheads $[6,9]$.

Since the error probability of a data burst depends on the channel conditions to the subscribers, the probability of successful transmission can be improved by distributing slots of each data burst into different time of slots and subchannels. Therefore, this paper proposes a scattered mapping method called fault-tolerant transmission mechanism (FTM) by breaking up the burst blocks into slots and then allocating them into different positions in the frame via a turntable algorithm (TA). By using FTM, the slots of previous bursts can be rearranged and will be put into the whole frame space to support a stable and secure transmission. A detailed FTM will be illustrated in following sections.

The remainder of this paper is organized as follows: Section 2 takes an overview of IEEE 802.16e scheduled transmission and illustrates our system model. Section 3 describes FTM in detail to improve the fault tolerance ability at MAC layer. Section 5 describes the implementation of the proposed mechanism with simulation results. Finally, the conclusion and the future works are discussed in Section 6 .

\section{SYSTEM MODEL}

Assume multiple MSSs are connected to a centralized BS over wireless fading channels, where multiple connections (data flows) can be supported by each MSS. All connections communicate with the BS using time division multiplexing/time division multiple access (TDM/TDMA). A buffer is implemented at the BS for each connection and operates in a first-input-first-output (FIFO) mode. The adaptive modulation and coding (AMC) controller follows the buffer at the BS (transmitter), and the AMC selector is implemented at the MSS (receiver). Each connection employs AMC scheme at the PHY layer.

Based on IEEE 802.16e OFDMA specifications [4], the operating spectrum can be divided into $N_{c}$ subchannels and each subchannel occupies $N_{s}$ time slots for multiple access usage as shown in Fig. 1. The modulation scheme quadrature phase-shift keying (QPSK), $M_{n}$-ary rectangular/square quadrature amplitude modulators (QAMs), the forward error correction (FEC) codes, and Reed-Solomon (RS) concatenated with convolutional codes (CC) schemes are considered.

Since the wireless channel quality is mainly subject to the instantaneous signal-to-noise ratio (SNR) $\gamma$, which is the statistical description based on the general Nakagami$m$ model $[1,11]$, the received SNR $\gamma$ per frame can thus be a random variable with a Gamma probability density function, i.e.,

$$
p_{\gamma}(\gamma)=\frac{m^{m} \gamma^{m-1}}{\bar{\gamma}^{m} \Gamma(m)} \exp \left(-\frac{m \gamma}{\bar{\gamma}}\right),
$$

where $m$ is the Nakagami fading parameter $(m \geq 1 / 2), \bar{\gamma}=$ $E\{\gamma\}$ is the average received SNR, and $\Gamma(m)=\int_{0}^{\infty} t^{m-1} e^{-t} d t$ is the Gamma function [2], respectively. This channel model is suitable for flat-fading channels as well as frequency-selective fading channels in the OFDMA system. This model includes the Rayleigh channel when $m=1$. Let $N_{m}$ denote the total number of transmission modes available. According to [1], the transmission power is assumed constant and the entire SNR range is partitioned into $N_{m}+1$ nonoverlapping consecutive intervals, with boundary points denoted as $\left\{\gamma_{n}\right\}_{n=0}^{N_{m}+1}$. In this case, mode $n$ is chosen when

$$
\gamma \in\left[\gamma_{n}, \gamma_{n+1}\right), \quad n=1,2, \ldots, N_{m},
$$

in which $\gamma$ should be in the range of the corresponding modulation and coding rate. To avoid deep-channel fading, no data are sent when $\gamma_{0} \leq \gamma<\gamma_{1}$, which corresponds to the mode $n=0$ with rate $R_{0}=0$ bits/symbol.

To simplify the AMC design, we approximate the slot error rate (SER) expression in AWGN channel as

$$
\operatorname{SER}_{n}(\gamma) \approx \begin{cases}1, & \text { if } 0<\gamma<\gamma_{p}^{n} \\ a_{n} \exp \left(-g_{n} \gamma\right), & \text { if } \gamma \geq \gamma_{p}^{n}\end{cases}
$$

where $n$ is the mode index and $\gamma$ is the received SNR. Parameters $a_{n}, g_{n}$, and $\gamma_{p}^{n}$ are mode-dependent and are obtained by fitting (3) to the exact SER via simulations presented in [10]. Let the region boundary (switching threshold) $\gamma_{n}$ for the transmission mode $n$ be the minimum SNR required to guarantee $S_{0}$. Inverting the SER expression in (3), we obtain

$$
\begin{aligned}
\gamma_{0} & =0 \\
\gamma_{n} & =\frac{1}{g_{n}} \ln \left(\frac{a_{n}}{S_{0}}\right), \quad n=1,2, \ldots, N_{m} \\
\gamma_{N+1} & =+\infty,
\end{aligned}
$$

By using (4) to specify $\left\{\gamma_{n}\right\}_{n=0}^{N_{m}}$, one can verify that the AMC in (2) guarantees that the SER is less than or equal to $S_{0}$.

Let $P_{i}(\gamma)$ be the successful transmission probability of a time slot in the $i$ th subchannel, where $i=0,1, \ldots, N_{c}-1$ is the subchannel index in the frame space. For simplicity, we assume the successful transmission probabilities with modulation mode $n$ of time slots are equal if they are in the same subchannel. Then $P_{i}(\gamma)$ can be expressed by using (3) as

$$
P_{i}(\gamma) \approx \begin{cases}0, & \text { if } 0<\gamma<\gamma_{p}^{n} \\ 1-a_{n} \exp \left(-g_{n} \gamma\right), & \text { if } \gamma \geq \gamma_{p}^{n}\end{cases}
$$

Thus the successful transmission probability of $k$ consecutive time slots in the $i$ th subchannel can be simply obtained by

$$
P_{i}(\gamma)^{k}=\underbrace{P_{i}(\gamma) P_{i}(\gamma) \cdots P_{i}(\gamma)}_{k \text { time slots }}
$$

Since a data burst consists of several MAC protocoal data units (MPDUs) and may occupies several subchannels for 


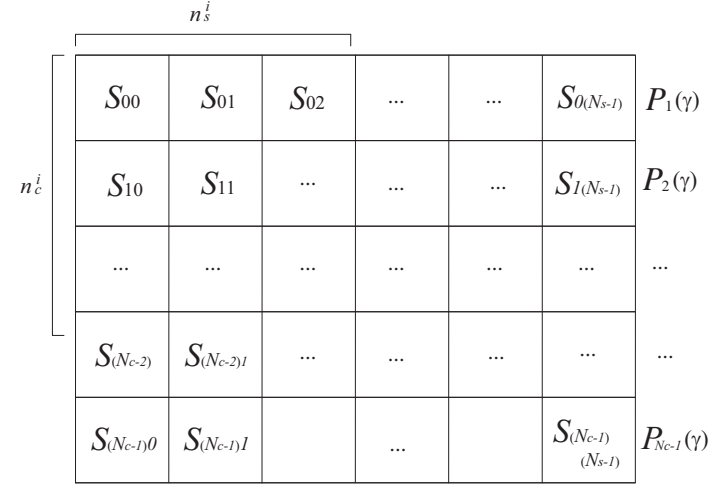

Figure 2: The structure of slot.

data transmission, see Fig. 1, the MPDU successful probability can be expressed as $P_{i}(\gamma)^{N_{b}}$, where $N_{b}=L / R_{n}$ represents the needed number of time slots to convey the MPDU, $L$ (bits) is the length of the MPDU, and $R_{n}$ is the data rate with modulation and coding mode $n$.

However, an MPDU will be allocated across several subchannels as it is long. Thus, a general equation of the successful transmission probability of an MPDU is as

$$
P_{d}(\gamma)=\underbrace{P_{i}(\gamma) \cdots P_{i}(\gamma)}_{s_{i} \text { time slots }} \underbrace{P_{i+1}(\gamma) \cdots P_{i+1}(\gamma)}_{s_{i+1} \text { time slots }} \cdots,
$$

where $i \in\left[0, N_{c}-1\right]$ is the beginning subchannel of carrying the MPDU and $N_{b}=s_{i}+s_{i+1}+\ldots+s_{i+k-1}$ for $k$ number of subchannels. Note that $P_{i}(\gamma)$ may vary from subchannel to subchannel depending on received $\gamma$ and its interferences.

Since each burst $B_{i}$ consists of several MPDUs and its slot allocation should be taken in rectangular basis (in the downlink period), the successful transmission probability of $B_{i}$ denoted as $P_{s}\left(B_{i}\right)$ could be calculated as

$$
P_{s}\left(B_{i}\right)=\prod_{j=0}^{n_{m}^{i}-1} P_{d}^{j}(\gamma)=\prod_{j=k}^{k+n_{c}^{i}-1}\left(P_{j}(\gamma)\right)^{n_{s}^{i}},
$$

where $n_{m}^{i}$ represents the total number of MPDUs in $B_{i}, k$ represents the starting subchannel of $B_{i}, n_{c}^{i}$ indicates the number of subchannels, and $n_{s}^{i}$ is the number of slots in each subchannel of $B_{i}$. The frame structure of the time slot is illustrated in Fig. 2.

In the subchannel, the distribution of the probability is defined as the normal distribution. The original probability $P_{s}\left(B_{i}\right) \in[0,1]$, which can be applied to the slots in the corresponding subchannel. To estimate other situations, such as the serious interference environment, the specific subchannels are subject to the fading effect [1]. The model designs the adjusted probability $P_{i}^{A}$ by

$$
P_{i}^{A}=P_{i}(\gamma) \bmod P_{U}+P_{L},
$$

where $P_{U}$ and $P_{L}$ are the adjusted successful transmission probability of the constraint upper and lower bound, respectively.

\section{FAULT-TOLERANT TRANSMISSION MECH- ANISM}

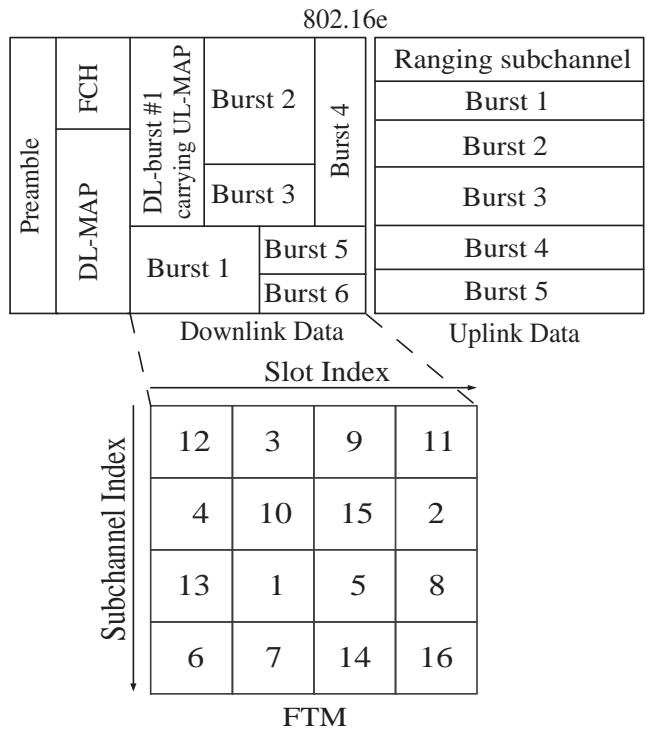

Figure 3: An example of time slot rearrangement by FTM.

The FTM is achieved by adjusting the time slot allocation of $B_{i}$ with each other. The reason is that each subchannel has its corresponding successful transmission probability $P_{i}(\gamma)$. The failed transmission probability caused by unpredictable external interference can be dispersed to different $B_{i}$, if some subchannel is under interference. Fig. 3 shows an example of the rearranged time slots of burst blocks in the downlink frame period. This mechanism can be applied to the permutations in some formula. Assume there are $N=N_{c} N_{s}$ time slots in the downlink subframe. The permutation group $G$ can be formulated as

$$
G=\left(\begin{array}{ccccc}
1 & 2 & \ldots & N-1 & N \\
13 & 20 & 5 & \ldots & \ldots
\end{array}\right)
$$

where the first row indicates the order of the original permutation of the time slots and the second row indicates the new arranged permutation of original time slots. This rearrangement is an one-to-one correspondence mapping. The time $T(G)$ with the product of the permutation can be modeled as

$$
T(G)=\frac{1}{|G|} \sum_{g \in G} \prod_{k=1}^{n} a_{k}^{j_{k}(g)},
$$

where $|G|$ and $g \in G$ are the absolute time of the permutation and a time of permutation, respectively; $k$ and $j_{k}(g)$ are the length of the permutation cycle and the number of cycles with the permutation cycle length $k$ in $g$ [13].

The details of probability-based FTM are described in the following four steps.

- STEP $1 P_{s}\left(B_{i}\right)$ Computing: First FTM obtains the successful transmission probability $P_{s}\left(B_{i}\right)$ for all $i=0,1, \ldots, N_{B}-1$, where $N_{B}$ is the number of bursts in this time period by using (8).

- STEP 2 Turntable Algorithm Optimal Choice: The problem of determining the optimal slot allocation from $G$ among $B_{i}$ so that the average successful 


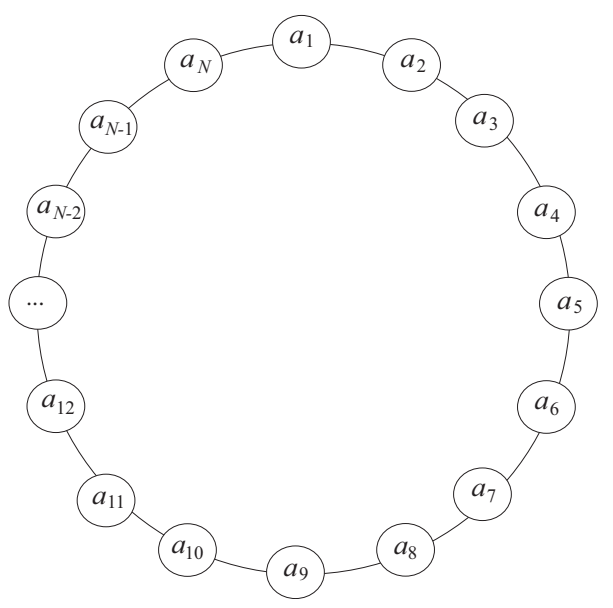

Figure 4: The rotational diagram of turntable structure in FTM.

transmission probabilities of all $B_{i}$ denoted as $P_{s}^{\prime}(t)=$ $\sum_{i=0}^{N_{B}-1} P_{s}^{\prime}\left(B_{i}\right) / N_{B}$, where $P_{s}^{\prime}\left(B_{i}\right)$ is the successful transmission probability of rearranged $B_{i}$, is greater than $P_{s}(t)=\sum_{i=0}^{N_{B}-1} P_{s}\left(B_{i}\right) / N_{B}$, or $P_{s}^{\prime}(t) \geq P_{s}(t)$, is an NP-complete problem [13]. To obtain a better $P_{s}\left(B_{i}\right)$, the time slots is aligned according to their corresponding $P_{i}(\gamma)$ in descending order, $P\left(S_{i j}\right) \geq P\left(S_{k l}\right)$ for all $i, k=0,1, \ldots, N_{c}-1$ and $j, l=0,1, \ldots, N_{s}-1$, where $P\left(S_{i j}\right)$ denotes the successful transmission probability of slot $S_{i j}$, and circle this order by letting the last slot follow the first slot as shown in Fig. 4. We note that $P\left(S_{i j}\right)=P_{i}(\gamma)$ since it is in the $i$ th subchannel.

- STEP 3 Dynamic Rotational Speed Estimating: Let $a_{1}, a_{2}, \ldots, a_{N}$ denote the aligned time slots in the turntable and $a_{1}=1, a_{2}=2, \ldots, a_{N}=N$. The time slot is assigned to $B_{i}$ by rotating the turntable of the descending order slots. The rotational speed $V_{R} \in \mathbb{N}$ is used to control the number of shifts of the turntable for selecting the next slot. For example, when $V_{R}=1$, $B_{i}$ will select $a_{1}, a_{2}, a_{3}, \ldots$ or when $V_{R}=3, B_{i}$ will pick the slot index on $a_{1}, a_{4}, a_{7}, \ldots$, and so on if the starting slot is $a_{1}$. The selection can be achieved by using modulo function, it follows

$$
a_{j}=a_{j-1}+V_{R} \equiv 0\left(\bmod V_{R}\right) .
$$

The burst $B_{i}$ with higher $P_{s}\left(B_{i}\right)$ will have the priority to select slots than others in the turntable. The $V_{R}$ will be increased by 1 when $B_{i}$ finishes its selection of $a_{j}$. If the indicated $a_{j}$ has been selected by other $B_{i}$ the index $j$ will be increased 1 until an unselected $a_{k}$ is found. Once $a_{j}$ has been selected, $a_{j}$ will be removed from the turntable immediately.

- STEP $4 P_{s}^{\prime}\left(B_{i}\right)$ Calculation: After scattering time slots, the $P_{s}^{\prime}\left(B_{i}\right)$ of each $B_{i}$ is calculated by

$$
P_{s}^{\prime}\left(B_{i}\right)=\prod_{j \in\left[0, N_{c}-1\right]} \prod_{k \in\left[0, N_{s}-1\right]} P\left(S_{j k}\right),
$$

where $S_{j k}$ is the selected slots by $B_{i}$.

\subsection{The Modification of MAC Layer}

The modification of this proposed mechanism is done by the embedded mechanism in MAC Layer of IEEE 802.16e. Hence, FTM is fully compatible with IEEE 802.16e standard without any other adjustment needed in the hardware. The only change is the rule of transmission. The following statement is used for illustrating the changing strategy. Basically, FTM is built in MAC Layer of BS and periodically operated by the BS or by the request of MSSs. When FTM of BS breaks all slots and distributes them into the whole frame space, it will produce several mapping patterns. These mapping patterns of FTM will be stored in BS reserved space in DL-MAP and will be used for a while. When a MSS successfully register and get the bandwidth grant from BS, BS produces several mapping patterns of FTM. The BS broadcasts these mapping patterns by DL-MAP MAC message and informs MSS of the correct mapping patterns to use. BS can handle many mapping patterns of FTM. MSSs will keep these patterns, which will be used when MSSs successfully connect with BS.

There are additional advantages in using FTM: security and confidentiality in BWA system. In some situations, FTM can be transmitted in security. Only MSSs can register at the BS by its own CID and receive valid mapping pattern. Therefore, this environment provides a new secure service, called the level of security. On the other hand, if some invaders want to wiretap or steal information in the current BWA system by air, by probing and collecting the specification of BS, the invader will get the system information without being able to handle these patterns.

In addition, the information of frame space will be changed periodically. Accordingly, the distributed scattered mapping patterns would not be duplicated. Although the invader understands the recombination of the frame by FTM, this information is invalid as time goes by. BS controls and changes the scattered mapping pattern. If the channel condition changes often, BS will be aware of the dramatic changing of CINR in the subchannel and the system will apply FTM again; the invader would have no time to deal with the continuous frames of the system. Moreover, if the invader use the force method, it should be extremely difficult to find the correct information of the frame space.

The transmission mechanism of IEEE $802.16 \mathrm{e}$ standard is based on bursts. Accordingly, to control the bursts is to dominate IEEE 802.16e system. FTM manages the burst size and allocates transmission slots. Therefore, the level of security is created by changing the probability distribution of all subchannels and adjusting the range of the probability value to express the whole channel quality. In these ways, FTM offers a vary safe transmission environment.

\subsection{An Example of Slots Selection}

In this section, the way in which the TA selects bursts slots will be demonstrated. For comparison, an example of the turntable algorithm is taken as shown in Fig. 5. Assume that there are six subchannels, four slots in each subframe, and six burst blocks as defined as the original burst blocks.

The FTM computes the $P_{s}\left(B_{i}\right)$ of each block to decide the sequence of each burst. The $P_{s}\left(B_{i}\right)$ of each burst block is listed in Table 1. According to $P_{s}\left(B_{i}\right)$, the the sequence of bursts is $(\mathrm{B}),(\mathrm{C}),(\mathrm{E}),(\mathrm{F}),(\mathrm{A})$, and (D). These six bursts will be orderly operated in the turntable algorithm. In burst (B) two calculated slots are chosen. By using different ar- 


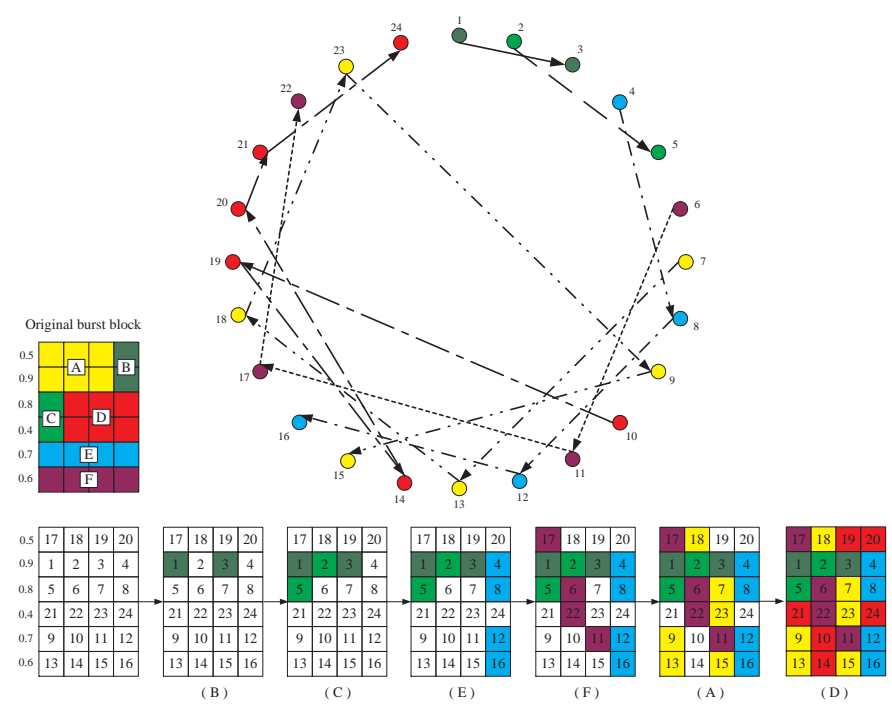

Figure 5: An example of FTM when the initial $V_{R}=$ 2 .

Table 1: Parameter of $P_{s}\left(B_{i}\right)$

\begin{tabular}{cc}
\hline Burst block index & $P_{s}\left(B_{i}\right)$ \\
\hline A & 0.091125 \\
B & 0.45 \\
C & 0.32 \\
D & 0.032768 \\
E & 0.2401 \\
F & 0.1296 \\
\hline Average probability & 0.2106 \\
\hline
\end{tabular}

rows, the TA points out the selection of bursts. Each burst has one kind of arrow. The miscellaneous arrows means the different bursts. These arrows also point to the index of slots, which represents bursts. Under the Fig. 5 shows the order of each burst entering TA.

After all bursts are calculated by the TA, the BS will compute the $P_{s}\left(B_{i}\right)$ for current burst. Table. 2 lists parameters of $P_{s}^{\prime}\left(B_{i}\right)$. Compared with the corresponding burst of Table. 1 , the average $P_{s}^{\prime}\left(B_{i}\right)$ is obvious to be higher than the original burst block.

\section{PROOF AND ANALYSIS}

The goal of FTM is to make majority user have greater $P_{s}\left(B_{i}\right)$ than the original one and the proof of this goal is illustrated in this section. Based on the NP-Complete problem, the effect and advantage of FTM will be estimated as follows:

Proposition 1: Prove the result of the FTM to be better than the greedy solution (GS) in the slotted mapping problem. The GS finds the best situation during the procedure. Hence, GS only concerns the current situation.

Proof. Let $P_{i}$ be the successful probability for each user after being processed by the FTM. The slot of $P_{i}$ is

$$
P_{i}=\left(S_{i 1}, S_{i 2}, \ldots, S_{i n}\right) .
$$

The successful probability of next user is $P_{i+1}$ and $P_{i} \geq$ $P_{i+1}$. Assume that there is another $P_{j}$ and $P_{j} \geq P_{i}$. The
Table 2: Parameter of $P_{s}^{\prime}\left(B_{i}\right)$

\begin{tabular}{cc}
\hline Burst block index & $P_{s}^{\prime}\left(B_{i}\right)$ \\
\hline A & 0.04032 \\
B & 0.81 \\
C & 0.72 \\
D & 0.0168 \\
E & 0.3024 \\
F & 0.112 \\
\hline Average probability & 0.3336 \\
\hline
\end{tabular}

$P_{j}$ also has several slots as

$$
P_{j}=\left(S_{j 1}, S_{j 2}, \ldots, S_{j n}\right) .
$$

For the assumption $P_{j} \geq P_{i}$, it must have

$$
\sum_{\alpha=1}^{n} P\left(S_{j \alpha}\right) \geq \sum_{\alpha=1}^{n} P\left(S_{i \alpha}\right)
$$

If the numbers of the slots are $S_{i 1}, S_{i 2}, \ldots, S_{i n}, \ldots, S_{i j}$, and the probability of $S_{i(n+1)}, S_{i(n+2)}, \ldots, S_{i j}$ are greater than $S_{i 1}, S_{i 2}, \ldots, S_{i n}$, then $P_{j} \geq P_{i}$ can be found. But in the predefinition, $\forall S_{x y}$, the slot $S_{i(n+1)}, S_{i(n+2)}, \ldots, S_{i j}$ are used by other users at the same time. In the FTM, the slot with the largest value will be chosen as the first slot by each user. The second slot is chosen by each user, whose value may be equal to the first one or be in the second place. Assume there is a solution, and the total probability of its user is greater than FTM. Then, the largest value of a slot in the system must always be chosen. This is so called the greedy solution. The calculating of GS will be faster than that of FTM. In this solution there should be a largest slot, which competes in the same subchannel, and it will run out earlier than the FTM. Because the largest slots has run out first, it can be deduced that if $P_{j}>P_{i}$, and then $P_{j+1}<P_{i+1}$. Accordingly, that $P_{j} \nsucceq P_{i}$ is proved and so the effective value $P_{i}$ is also proved.

Proposition 2: Prove the probability of the condition of $P_{s}^{\prime}\left(B_{i}\right)>P_{s}\left(B_{i}\right)$ is high.

Proof. Suppose that one user needs $n$ slots. In the solution of IEEE 802.16e, a user can make a choice on $T$ slots. In the solution of FTM one user can make a choice on the total frame space. Specifically, one user can make a choice on $N$ slots, where $N>T$. Assume that the $P_{f}$ is the probability of $P_{s}\left(B_{i}\right)>P_{s}^{\prime}\left(B_{i}\right)$.

$$
\begin{aligned}
P_{f} & =\frac{\left(\begin{array}{l}
T \\
n
\end{array}\right)}{\left(\begin{array}{l}
N \\
n
\end{array}\right)} \\
& =\frac{\frac{T !}{n !(T-n) !}}{N !} \\
& =\frac{T !(N-n) !}{(T-n) ! N !} \\
& =\frac{(N-n)(N-n-1) \ldots(T-n+1)}{N(N-1) \ldots(T+2)(T+1)}
\end{aligned}
$$


Table 3: Simulation Parameter

\begin{tabular}{lc}
\hline Parameter & Value \\
\hline Frame length $(\mathrm{ms})$ & 5 \\
FFT size $\left(N_{F F T}\right)$ & 1024 \\
Bandwidth $(\mathrm{MHz})$ & 10 \\
Sampling frequency $\left(F_{s}\right)(\mathrm{MHz})$ & 11.42 \\
Subcarrier spacing $\left(\Delta f=F_{s} / N_{F F T}\right)(\mathrm{KHz})$ & 11.16 \\
Cyclic prefix time $\left(T_{g}\right)(\mu \mathrm{s})$ & 11.20 \\
Useful symbol time $\left(T_{b}\right)(\mu \mathrm{s})$ & 89.64 \\
OFDMA symbol time $\left(T_{s}\right)(\mu \mathrm{s})$ & 100.84 \\
No. of OFDMA symbol per frame & 49 \\
TTG, RTG $(\mu \mathrm{s})$ & 29.41 \\
No. of OFDMA symbol per frame for data & 48 \\
No. of OFDMA symbol per frame for downlink data & 36 \\
No. of OFDMA symbol per frame for uplink data & 12 \\
No. of OFDMA downlink subchannel & 30 \\
\hline
\end{tabular}

where

$$
\overbrace{\frac{(N-n)(N-n-1) \ldots(T-n+1)}{\underbrace{N(N-1) \ldots(T+2)(T+1)}_{N-T}}}^{N-T} .
$$

Because of the number of numerator and the number of denominator are equal. These two sequence numbers are descending, each element in each index can be compared. For example, if $N>(N-n),(N-1)>(N-n-1), \ldots$, $(T+1)>(T-n+1)$, then the denominator is greater than the numerator in each element. Furthermore, if $N \gg T$, then it is obvious that denominator $\gg$ numerator. This implies that $P_{f} \ll 1$. Therefore, that $P_{s}^{\prime}\left(B_{i}\right)>P_{s}\left(B_{i}\right)$ has been proved.

\section{SIMULATION MODEL AND RESULTS}

In order to evaluate IEEE 802.16e and our transmission mechanism, a ns2-simulation model is designed to simulate successful transmission probability and system throughput during the transmission process [3]. IEEE 802.16e MAC and PHY protocol are adopted as the data link layer and the physical layer, respectively. The probability for slots distributed to be each subchannel is a normal distribution. The bandwidth of one channel is $10 \mathrm{MHz}$ in OFDMA-TDD mode and the frame length is $5 \mathrm{~ms}$. The simulation model is a only performed in the downlink subframe. There are 30 subchannels and 36 OFDMA symbols of data. OFDMA data slot mapping mode is partial usage of subchannels, in which one slot includes two OFDMA symbols. In the PUSC mode there are 18 slots in the DL subframe. The channel encoding type is convolutional coding (CC). This model considers all MSSs use the same modulation/coding type. The simulation model run at 30 times to get the average value in one channel. The simulation model-specific parameters are listed in Table 3.

Fig. 6 compares the relation between the maximum burst size $\left|B_{i}\right|$ and $P_{s}(t)$ in IEEE 802.16e and $P_{s}^{\prime}(t)$ in FTM. $\left|B_{i}\right|$ is limited to 1 to 200 time slots in the downlink frame space. This result shows that both $P_{s}(t)$ and $P_{s}^{\prime}(t)$ decrease when $\left|B_{i}\right|$ increases. It is obvious to understand that the larger the $\left|B_{i}\right|$ is the unsuccessful transmission probability will increase. However, it is interest to observe that FTM outperforms IEEE 802.16e in $P_{s}^{\prime}(t)>P_{s}(t)$ around 2 to 5 times. The reason why FTM outperforms IEEE $802.16 \mathrm{e}$ is that FTM by scrambling time slots of each $B_{i}$ averages the single

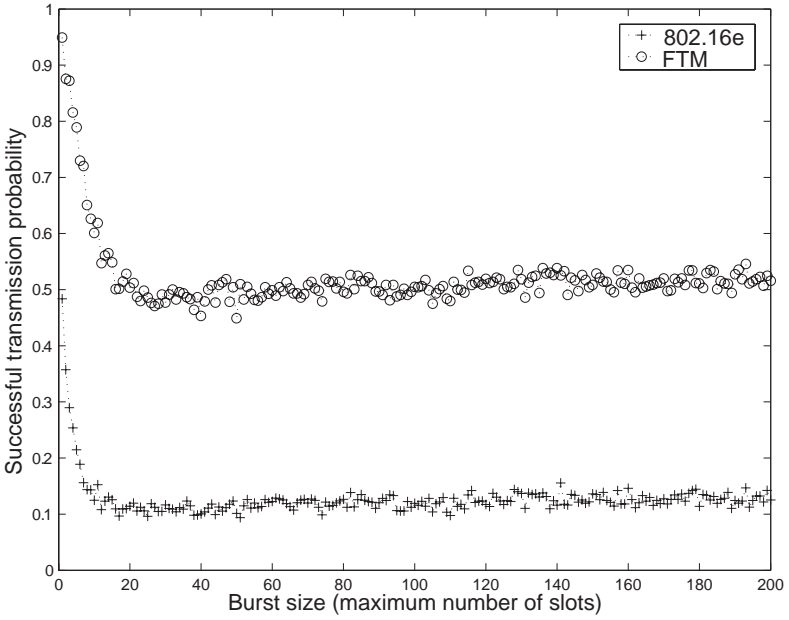

Figure 6: The successful transmission probability vs. $M$ (maximum number of slots).

subchannel failed possibility to increase $P_{s}^{\prime}(t)$. Hence, from this result, it shows that an efficient burst size should be as small as no more than 20 slots or $\left|B_{i}\right|$ should not exceed $5 \%$ of the downlink frame space.

To measure the system throughput, the valid burst $B_{i}^{V}$ is defined as it has enough slots to handle the data transmission. The throughput indicates $B_{i}^{V}$ of the corresponding modulation. If the required transmission slots exceeds the maximum valid bursts in the assigned channels, the data will not be transmitted and its throughput will decrease. The channel encoding type uses convolutional coding. Fig. 7 illustrates an example of our simulations and the simulation modulation parameters are 64-QAM 3/4 $\gamma=$ $17.9629 \mathrm{~dB}, 27$ bytes/slot. The throughput of IEEE $802.16 \mathrm{e}$ and FTM is in the condition of 64 -QAM $3 / 4$ and is limited by $P_{i}(\gamma) \in[0.5,1]$. When $N_{B}=100$, the throughput of FTM reaches $18.1 \mathrm{Mbps}$ than that of IEEE 802.16e reaches 15.5 Mbps. The throughput of simulation results are listed in Table 4. From these results, we emphasized that the transmission efficiency will be achieved when the fact that the higher modulation will lead to higher valid data payload is taken into consideration. However, a higher modulation will need a higher SNR to transmit and to receive. The higher modulation will bring about higher throughput and will need better channel quality and vice versa.

\section{CONCLUSION}

In this paper, we propose a fault-tolerant transmission mechanism (FTM), which periodically adjusts mapping patterns, for BS to control the burst size and allocates transmission slots. The simulation results illustrate that FTM can yield approximate $30 \%$ throughput and significantly raise the successful transmission probability, compared to IEEE 802.16e transmission mechanism. Accordingly, a conclusion can be reached that the burst size should be restricted no more than 20 slots even under the assigned maximum burst size; a burst size should not exceed $5 \%$ of the total frame space; a higher modulation will produce larger throughput and need better channel quality and vice versa. In addition, in our proposed solutions, FTM is a dynamic solution, which can serve all frame-based system and is ready 


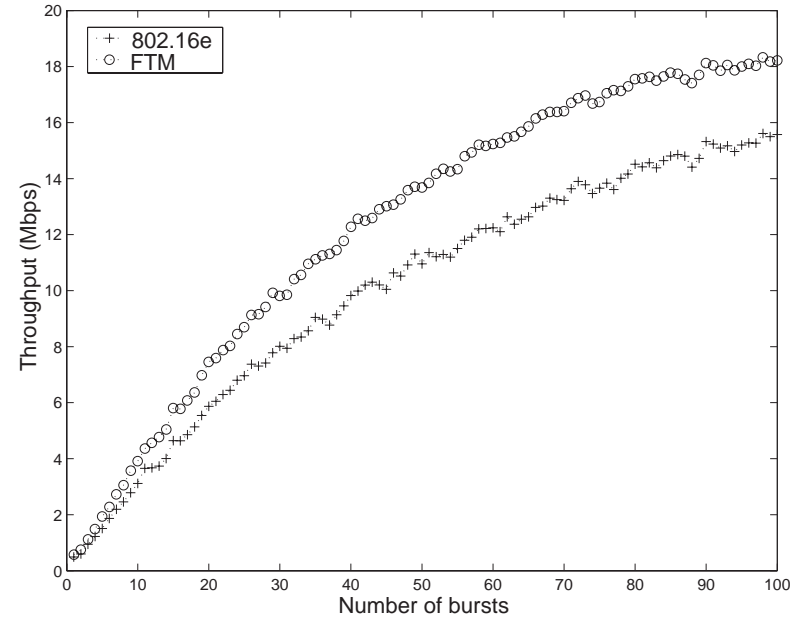

Figure 7: The comparison of throughput of IEEE 802.16e and FTM vs. $N_{B}$ with $\gamma_{n}=64-$ QAM 3/4 and $P_{i}(\gamma) \in[0.5,1]$.

Table 4: Throughput Results

\begin{tabular}{|c|c|c|c|}
\hline Modulation & Mechanism & Limited $P$ & Throughput \\
\hline \multirow[t]{2}{*}{ QPSK-1/2 } & IEEE $802.16 \mathrm{e}$ & $\mathrm{NO}$ & $4.4 \mathrm{Mbps}$ \\
\hline & FTM & $\mathrm{NO}$ & $4.5 \mathrm{Mbps}$ \\
\hline \multirow[t]{2}{*}{ QPSK-3/4 } & IEEE $802.16 \mathrm{e}$ & $\mathrm{NO}$ & $6.25 \mathrm{Mbps}$ \\
\hline & FTM & NO & $6.5 \mathrm{Mbps}$ \\
\hline \multirow[t]{2}{*}{ 16-QAM-1/2 } & IEEE $802.16 \mathrm{e}$ & NO & $7.8 \mathrm{Mbps}$ \\
\hline & FTM & $\mathrm{NO}$ & $8.8 \mathrm{Mbps}$ \\
\hline \multirow[t]{2}{*}{ 16-QAM-3/4 } & IEEE $802.16 \mathrm{e}$ & $\mathrm{NO}$ & 11.9Mbps \\
\hline & FTM & NO & $12.8 \mathrm{Mbps}$ \\
\hline \multirow{2}{*}{ 64-QAM-2/3 } & IEEE $802.16 \mathrm{e}$ & $\mathrm{NO}$ & $9.8 \mathrm{Mbps}$ \\
\hline & FTM & $\mathrm{NO}$ & $11.9 \mathrm{Mbps}$ \\
\hline \multirow[t]{6}{*}{ 64-QAM-3/4 } & IEEE $802.16 \mathrm{e}$ & $\mathrm{NO}$ & 8.9Mbps \\
\hline & FTM & $\mathrm{NO}$ & $9.8 \mathrm{Mbps}$ \\
\hline & IEEE $802.16 \mathrm{e}$ & $0.3-1$ & 10.9Mbps \\
\hline & FTM & $0.3-1$ & $13.1 \mathrm{Mbps}$ \\
\hline & IEEE $802.16 \mathrm{e}$ & $0.5-1$ & $15.5 \mathrm{Mbps}$ \\
\hline & FTM & $0.5-1$ & 18.1Mbps \\
\hline
\end{tabular}

to be disposed all over the existing network infrastructure. Hence, with transmission control in uplink, FTM can be investigated further in the future for supporting real-time QoS among macrocells.

\section{REFERENCES}

[1] M.S. Alouini, and A.J. Goldsmith, "Adaptive Modulation over Nakagami Fading Channels," Kluwer J. Wirel. Commun., vol. 13, no. 1/2, pp. 119-143, May 2000.

[2] J. Chen and W.-K. Tan, "Predictive Dynamic Channel Allocation Scheme for Improving Power Saving and Mobility in BWA Networks," ACM/Springer Mobile Networks and Applications (MONET), vol. 12, no. 1, pp. 15-30, February 2007.

[3] J. Chen, et al., "The Design and Implementation of WiMAX Module for ns-2 Simulator," in Proc. ACM VALUETOOLS 2006, vol. 202, article 5, Pisa, Italy, October 2006.

[4] IEEE 802.16 Working Group, "IEEE Standard for Local and Metropolitan Area Networks-Part 16: Air Interface for Fixed and Mobile Broadband Wireless
Access Systems," IEEE Std. 802.16e-2005, February 2006.

[5] M. El-Sayed and J. Jaffe, "A View of Telecommunications Network Evolution," IEEE Commun. Mag., vol. 40, no. 12, pp. 74-81, December 2002.

[6] A. Ghosh, D.R. Wolter, J.G. Andrews, and R. Chen, "Broadband Wireless Access with WiMax/802.16: Current Performance Benchmarks and Future Potential," IEEE Commun. Mag., vol. 43, no. 2, pp.129-136, February 2005.

[7] G. Kulkarni, S. Adlakha, and M. Srivastava, "Subcarrier Allocation and Bit Loading Algorithms for OFDMA-Based Wireless Networks," IEEE Trans. Mob. Computing, vol. 4, no. 6, pp. 652-662, November 2005.

[8] W.C.Y. Lee, "CS-OFDMA: A New Wireless CDD Physical Layer Scheme," IEEE Commun. Mag., vol. 43, no. 2, pp. 74-79, February 2005.

[9] R. Lee, J. Kim, J. Yu, J. Lee, and D. Kim, "Capacity Analysis Considering Channel Resource Overhead for Mobile Internet Access (WiBro)," IEEE VTC'05, vol. 5, pp. 3082-3086, May 2005.

[10] L. Li, FEC Performance with ARQ and Adaptive Burst Profile Selection, 2001.

[11] Q. Liu, X. Wang, and G.B. Giannakis, "A Cross-Layer Scheduling Algorithm With QoS Support in Wireless Networks," IEEE Trans. Vehicul. Technol., vol. 55, no. 3, pp. 839-847, May 2006.

[12] T. Pollet, M. Van Bladel and M. Moeneclaey, "BER Sensitivity of OFDM Systems to Carrier Frequency Offset and Wiener Phase Noise," IEEE Trans. Commun., vol. 43, no. 234, pp. 191-193, February 1995.

[13] J. Rotman, A First Course in Abstract Algebra, Prentice-Hall, 1996.

[14] H. Yaghoobi, "Scalable OFDMA Physical Layer in IEEE 802.16 WirelessMAN," Intel Technol. Journal, vol. 8, no. 3, pp. 201-212, August 2004.

[15] Y.H. You, B.J. Jang, and H.K. Song, "Low-Complexity and MAI-Robust Wireless Broadcasting System With Return Channel," IEEE Trans. Broadcasting, vol. 52, no. 1, pp. 71-76, March 2006.

[16] F. Ohrtman, WiMax Handbook-Building 802.16 Wireless Networks, McGrew-Hill, May 2005.

[17] T.J. Willink, P.H. Wittke, and L.L. Campbell, "Evaluation of the Effects of Intersymbol Interference in Decision-Feedback Equalizers," IEEE Trans. Commun., vol. 48, no. 4, pp. 629-636, April 2000. 